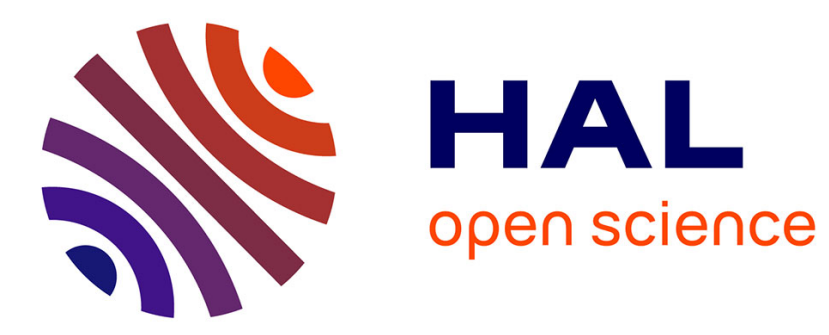

\title{
High resolution X-ray diffraction study of Smectic A - Smectic A phase transitions in a binary mixture of polar thermotropic liquid crystals
}

A.M. Levelut

\section{- To cite this version:}

A.M. Levelut. High resolution X-ray diffraction study of Smectic A - Smectic A phase transitions in a binary mixture of polar thermotropic liquid crystals. Journal de Physique Lettres, 1984, 45 (12), pp.603-611. 10.1051/jphyslet:019840045012060300 . jpa-00232388

\section{HAL Id: jpa-00232388 https://hal.science/jpa-00232388}

Submitted on 1 Jan 1984

HAL is a multi-disciplinary open access archive for the deposit and dissemination of scientific research documents, whether they are published or not. The documents may come from teaching and research institutions in France or abroad, or from public or private research centers.
L'archive ouverte pluridisciplinaire HAL, est destinée au dépôt et à la diffusion de documents scientifiques de niveau recherche, publiés ou non, émanant des établissements d'enseignement et de recherche français ou étrangers, des laboratoires publics ou privés. 
Classification

Physics Abstracts

$61.30 \mathrm{E}-64.70 \mathrm{M}$

\title{
High resolution X-ray diffraction study of Smectic A - Smectic A phase transitions in a binary mixture of polar thermotropic liquid crystals
}

\author{
A. M. Levelut \\ Laboratoire de Physique des Solides, Bâtiment 510, Université de Paris Sud, \\ 91405 Orsay Cedex, France \\ and \\ Laboratoire pour l'Utilisation du Rayonnement Electromagnétique (LURE), \\ Université de Paris Sud, 91405 Orsay Cedex, France
}

(Reçu le ler février 1984, accepté le 18 avril 1984)

\begin{abstract}
Résumé. - Utilisant le rayonnement synchrotron de LURE, nous avons étudié la séquence de phases mésogènes suivante : Smectique $A_{1}$ (couches monomoléculaires) - Antiphase fluide à réseau rectangle bidimensionnel - Smectique $A_{2}$ (couches bimoléculaires). Nous avons pu montrer qu'il peut exister deux phases en ruban à réseau rectangle de symétrie différente.

A la phase précédemment décrite $S_{\widetilde{A}}$ qui correspond à une alternance périodique, parallèlement aux couches de domaines antiphases d'égale importance mais de largeur croissant lorsque la température décroît, succède une seconde phase de période constante mais dans laquelle l'une des deux sortes de domaines antiphases décroît au profit de la seconde pour disparaitre complètement lorsqu'on atteint la phase Smectique $A_{2}$. La transition entre les deux phases bidimensionnelles s'accompagne d'un hystérésis thermique de plusieurs degrés.

Abstract. - With the help of the synchrotron radiation facility LURE, we have studied the following mesogenic phase sequence : Smectic $A_{1}$ (monolayered) - fluid antiphase with a rectangular 2D lattice - Smectic $A_{2}$ (bilayered), we have shown the existence of two ribbon phases with rectangular lattices of different symmetries. Besides the centred rectangular phase already described $\left(\mathbf{S}_{\tilde{\mathbf{A}}}\right)$ in which the two kinds of antiphase domains have an equal weight, we have found a second phase with a simple rectangular lattice in which the relative weight of the two kinds of domains varies with temperature until the $\mathrm{S}_{\mathrm{A}_{2}}$ phase is reached. In the centred rectangular phase, the in-layer periodicity increases with decreasing temperature while it remains constant in the simple rectangular phase. The transition temperature between these two phases exhibits a hysteresis of a few degrees.
\end{abstract}

It is now well established that the introduction of longitudinal electric dipoles on rod-like mesogenic molecules induces a rich polymorphism [1]. Let us consider Smectic A phases in which the molecules form a regular stack of infinite 2D liquid-like ordered layers with their director perpendicular to the layer, the presence of longitudinal dipoles raises the question of their orientation inside each layer. 
We have an $\mathrm{S}_{\mathrm{A}_{1}}$-phase when the dipoles are randomly oriented in each layer, the period is then equal to the molecular length. $\mathrm{A} \mathrm{S}_{\mathrm{A}_{2}}$-phase has a periodicity of twice the molecular length, therefore in this phase the dipoles are all in the same direction in each layer and are alternatively directed up and down in going from layer to layer.

The $\mathrm{S}_{\mathrm{A}_{2}}$-phase exists in the $\mathrm{DB}_{n}$ series :

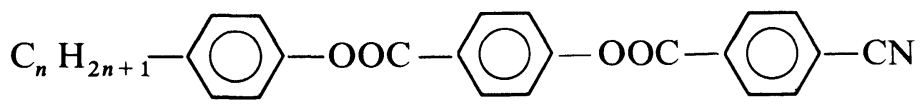

and the paraelectric-antiferroelectric transition was found for the first time in binary mixtures of $\mathrm{DB}_{5}$ with terephtalbisbutylaniline (TBBA) [2]. Binary mixtures of $\mathrm{DB}_{5}$ and $\mathrm{DB}_{6}$ with another mesogen $\mathrm{C}_{5}$-stilbene :<smiles>CCCCCc1ccc(C=C=Cc2ccc(OC(=O)c3ccc(C#N)cc3)cc2)cc1</smiles>

also presents these two phases but an intermediate state appears between the $S_{A_{1}}$ and $S_{A_{2}}$ phases for a limited range of concentration [3]. In this intermediate phase the orientation of the dipoles is periodically modulated in each layer while the antiparallel array subsists along the director. Therefore we can consider that this last phase is made up of antiphase domains, as seen in ordered alloys. These domains have a ribbon shape and form a regular centred rectangular phase.

This new phase was named $S_{\tilde{\mathbf{A}}}$ (fluid antiphase).

The sequence $S_{A_{1}}-S_{\tilde{A}}-S_{A_{2}}$ was followed by X-ray diffraction on oriented samples [3] : in the $\mathrm{S}_{\mathrm{A}_{1}}$-phase a unique Bragg spot appears at $q_{Z} \simeq \frac{2 \pi}{L}, q_{X}=0$

$Z$ is parallel to the direction,

$L$ is the molecular length.

When the temperature is lowered, diffuse spots appear at :

$$
q_{Z} \simeq \alpha \frac{2 \pi}{L} \quad q_{X} \neq 0.5<\alpha<1
$$

The $S_{\mathrm{A}_{1}}-\mathrm{S}_{\widetilde{\mathrm{A}}}$ transition corresponds to $\alpha \rightarrow 0.5$ and the diffuse spots become sharp at the same time.

In the $\mathrm{S}_{\tilde{\mathrm{A}}}$-phase, $q_{X}$ decreases and seems to go continuously to zero at the $\mathrm{S}_{\tilde{\mathrm{A}}}-\mathrm{S}_{\mathrm{A}_{2}}$ transition. Nevertheless, our resolution was not sufficient to allow a precise study of the two transitions. We report here new experiments done on mixtures of $\mathrm{DB}_{6}-\mathrm{C}_{5}$ with a molar concentration of $\mathrm{C}_{5}$ of about $55 \%$. We have performed X-ray diffraction experiments on aligned samples using synchrotron radiation and complete our results by powder patterns obtained with a small-angle scattering X-ray camera.

\section{Experiments.}

1.1 Aligned SAMPLES. - The samples were put in $1.5 \mathrm{~mm}$ Lindeman glass capillary tubes and aligned in the nematic phase with a $0.3 \mathrm{~T}$ magnetic field. The temperature of the sample was stable within $\pm 0.1 \mathrm{~K}$. The X-ray beam comes from the DCI synchrotron ring at LURE and was reflected by a $\mathrm{Ge}(111)$ bent single crystal. We used a wave length of $1.74 \AA$ with a $0.3 \mathrm{~mm}$ diameter collimator. The resolution was $\Delta q=3 \times 10^{-3} \AA^{-1}$ in a direction perpendicular to the director and $5 \times 10^{-3} \AA^{-1}$ in a direction parallel to the director.

We collected the X-ray diffracted intensity on photographic plates with a film-to-sample distance of $240 \mathrm{~mm}$. 
1.2 Powder Patterns. - The X-ray source was a Hilger microfocus tube with a cobalt anode $\left(\mathrm{CoK} \alpha_{1}=1.7889 \AA\right)$. We use a Johann Quartz monochromator followed by two sets of slits for limiting the scattering background. The sample is put in a Lindeman glass tube and the temperature stability is the same as for the single domain experiments. A photographic film is placed in the focussing plane of the monochromator at a distance of $179 \mathrm{~mm}$ from the sample. The resolution is $\Delta q=2 \times 10^{-3} \AA^{-1}$.

\section{Results.}

Figure 1 gives some examples of the $X$-ray patterns of the aligned samples. At high temperature in the $\mathrm{S}_{\mathrm{A}_{1}}$ - phase two Bragg spots appear at $q_{X}=0, q_{Z} \simeq \pm \frac{2 \pi}{L}= \pm q_{1}$ (Fig. 1a).

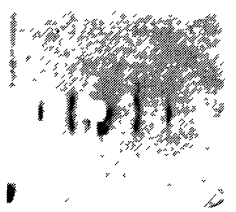

a

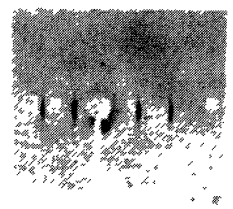

C

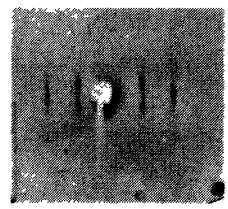

b

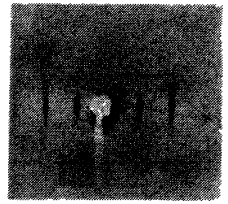

d

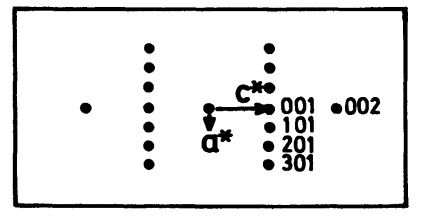

-

Fig. 1. - X-ray diffraction patterns of $\mathrm{DB}_{6} \mathrm{C}_{5}$ stilbene $55 \%$ obtained at LURE. a) $\mathrm{S}_{\mathrm{A}_{1}} 133{ }^{\circ} \mathrm{C}$; b) centred rectangular phase $131{ }^{\circ} \mathrm{C}$; c) rectangular phase $116^{\circ} \mathrm{C}$; d) $\mathrm{S}_{\mathrm{A}_{2}}$ phase $115^{\circ} \mathrm{C}$; e) schematic drawing of the reciprocal space $($ scale $\times 2$ ).

Four diffuse spots which are in fact the intersection of a ring with the Ewald sphere are progressively more visible when the $S_{\tilde{\mathbf{A}}}$ phase is approached. Following the position of these spots it is found that they are out of the $q_{X}=0$ axis at a nearly $q$ constant value; while $q_{Z}= \pm q_{2}$ decreases with temperature in such a way that the ratio $\alpha=q_{2} / q_{1}$ goes smoothly toward $1 / 2$ at the transition $S_{A_{1}}-S_{\tilde{A}}\left(132.5^{\circ} \mathrm{C}\right)$ (Fig. 2). The width of the diffuse spots also decreases with temperature (Fig. 3) and half a degree above the $S_{A_{1}}-S_{\tilde{A}}$ transition, one can estimate the correlation length for the modulation of the orientation of the dipoles, which are at the origin of the diffuse spots : $\xi_{2}>500 \AA$

$$
\xi_{1} \sim 120 \AA .
$$




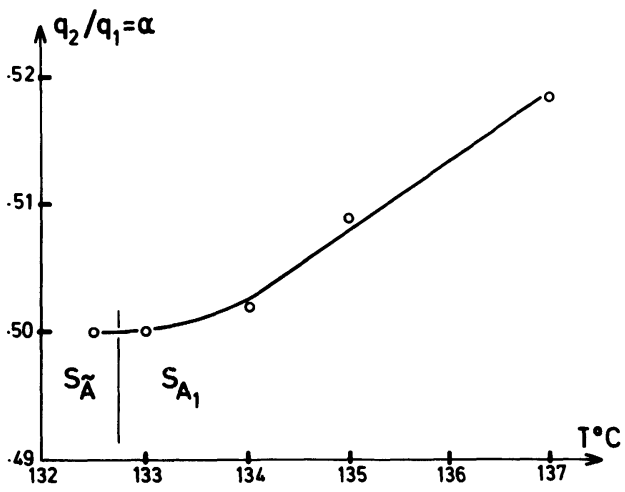

Fig. 2. - Temperature dependence of the ratio of the component along the director of the scattering vector of the diffuse spot versus the scattering vector of the Bragg spot in the $\mathrm{S}_{\mathrm{A}_{1}}$ phase.
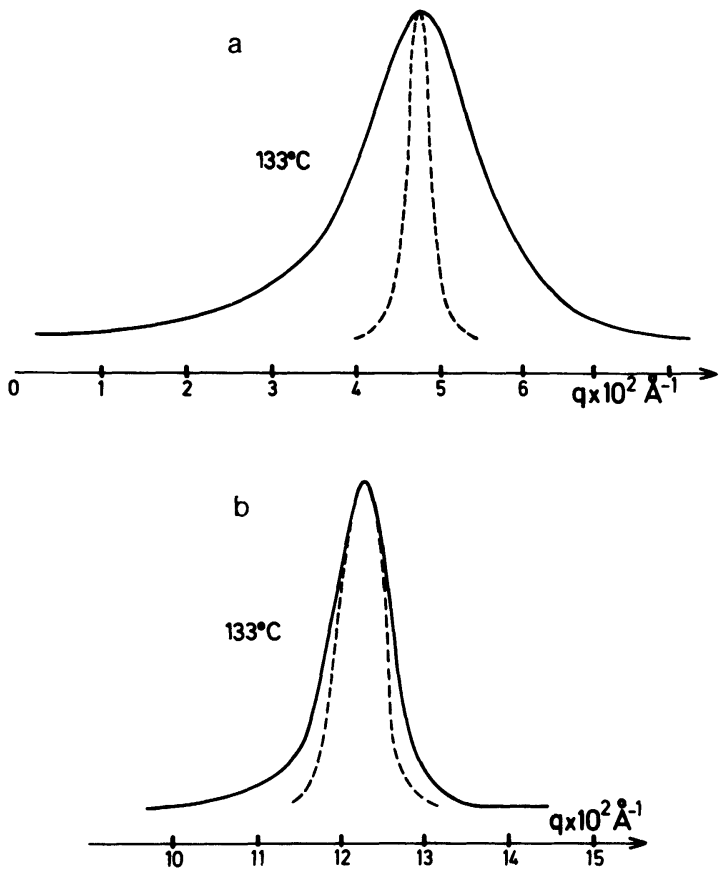

Fig. 3. - Densitometric scan of the diffuse spot seen in Fig. 1a, $T=133^{\circ} \mathrm{C}$; the dotted line shows the resolution function of the device measure on a harmonic $\lambda / 3$ Bragg reflection on smectic layers : a) along a direction perpendicular to the director; b) along a direction parallel to the director. The scale is given in $q=\frac{4 \pi \sin \theta}{\lambda}$ where $2 \theta$ is the scattering angle and $\lambda$ the wavelength.

$\xi_{2}$ is the correlation length in the direction of the director and in this direction the width of the spot is not much larger than the resolution.

$\xi_{1}$ measures the correlation length parallel to the layer planes.

In the $\mathrm{S}_{\widetilde{\mathrm{A}}}$ phase, we see Bragg spots at $q_{X}=0, q_{Z}= \pm q_{1}$ and $q_{X} \neq 0, q_{Z}= \pm q_{2}= \pm \frac{q_{1}}{2}$.

All the spots have the same width fixed by the resolution of the device. 
The diffraction pattern has a cylindrical symmetry around the director axis. There are domains of the centred rectangular phase with a common $\mathbf{c}$ axis and various directions of $\mathbf{a}$ in a plane perpendicular to $\mathbf{c}$.

In fact we can see on figure 1 that, besides domains with $\mathbf{c}$ parallel to the magnetic field, a small amount of other domains have their $\mathbf{c}$ axes slightly disoriented from this direction, and are at the origin of the striated appearance of the Bragg spots. We must also take notice of the fact that the Bragg spots are overexposed in order to observe diffuse scattering and higher order reflections of very low intensities. The lattice constants of the $S_{\tilde{\mathbf{A}}}$ phase are :

$$
c=\frac{2 \pi}{q_{Z}} \quad a=\frac{2 \pi}{q_{X}} .
$$

$c$ is almost constant over the whole temperature range of the $\mathrm{S}_{\tilde{\mathrm{A}}}$ phase while $a$ increases as we approach the transition toward the next phase seen at lower temperature. The number of visible reflections with $l=1$ increases also as the temperature decreases : 101 is visible in the whole range while the 301 point becomes visible (but with low intensity) (Fig. 1b) when we decrease the temperature. Therefore the dipolar orientation of dipoles is harmonic at high temperature and becomes more complex as we approach the $\mathrm{S}_{\mathrm{A}_{2}}$ phase. At $116^{\circ} \mathrm{C}$, on cooling a change in the diffraction pattern occurs : a spot appears on the $Z$ axis corresponding to the 001 reflection. At the same time, 301 reflections disappear and 201 (Fig. 1c) reflections are visible with a very weak intensity. At $115^{\circ} \mathrm{C}$ all Bragg spots lying out of the $Z$ axis disappear and we have the $S_{\mathrm{A}_{2}}$ phase (Fig. 1d). The pattern observed at $116^{\circ} \mathrm{C}$ is characteristic of a new two-dimensional phase with a noncentred rectangular lattice of size similar to that of the $S_{\tilde{\mathbf{A}}}$ phase. We must underline that the presence of 201 reflections allows us to exclude the possibility of having coexistence of $\mathrm{S}_{\mathrm{A}_{2}}$ and $\mathrm{S}_{\tilde{\mathrm{A}}}$. On heating the sample this second rectangular phase is stable between $115.5^{\circ} \mathrm{C}$ and $117.5^{\circ} \mathrm{C}$. The lattice constants remain unchanged but the intensities of the different Bragg spots vary. The peaks corresponding to $h 0 l$ reflections with $h+l=2 n$ increase with temperature while the peaks with $h+l=2 n+1$ decrease in intensity. Figure 4 shows microdensitometer scans along a line perpendicular to the magnetic field and going through the $h 01$ peaks. 101 and 001 peaks are out of scale but we can follow between $115.5^{\circ} \mathrm{C}$ and $118^{\circ} \mathrm{C}$ the evolution of the 201 and 301 peaks. We have noted a difference between the in-layer periodicity of the $S_{\tilde{A}}$ phase at the same temperature obtained either by heating the $S_{A_{2}}$ phase or by cooling the $S_{A_{1}}$ phase. In small angle diffraction experiments on powder samples, we have confirmed this hysteresis effect but we have not been able to detect the second rectangular phase because of the lack of resolution (Fig. 5). Another sample which contains a slightly lower amount of $\mathrm{C}_{5}$ molecules was also studied in powder samples and showed a smaller hysteresis for the $S_{\widetilde{A}}$ phase. This fact suggests that the second rectangular phase could disappear before the $S_{\tilde{\mathrm{A}}}$ phase with increasing concentration of $\mathrm{DB}_{6}$.

\section{Discussion.}

We have experimental evidence of the existence of another 2D ribbon rectangular phase between the $S_{\tilde{A}}$ and $S_{\mathbf{A}_{2}}$ phases. We will first recall the structure of the $S_{\tilde{\mathbf{A}}}$ phase before discussing the organization of this second phase.

The $S_{\tilde{\mathbf{A}}}$ phase has a centred rectangular lattice and therefore we have to consider that the same structural elements lie at the corner and at the centre of the rectangle. If we take into account the lattice spacing each structural element is a ribbon of thickness $c$, width $a / 2$ and equivalent infinite length. As the thickness equals twice the molecular length, the ribbon is thus a double layer of molecules of finite width in which the molecular director is perpendicular to the layer plane and the dipoles have an antiparallel configuration with let us say the nitrile groups in the middle plane and the chain at the two surfaces. Stacking these ribbons on a rectangular lattice keeps the layer structure unchanged with a periodicity of one molecular length, but at each interface we 

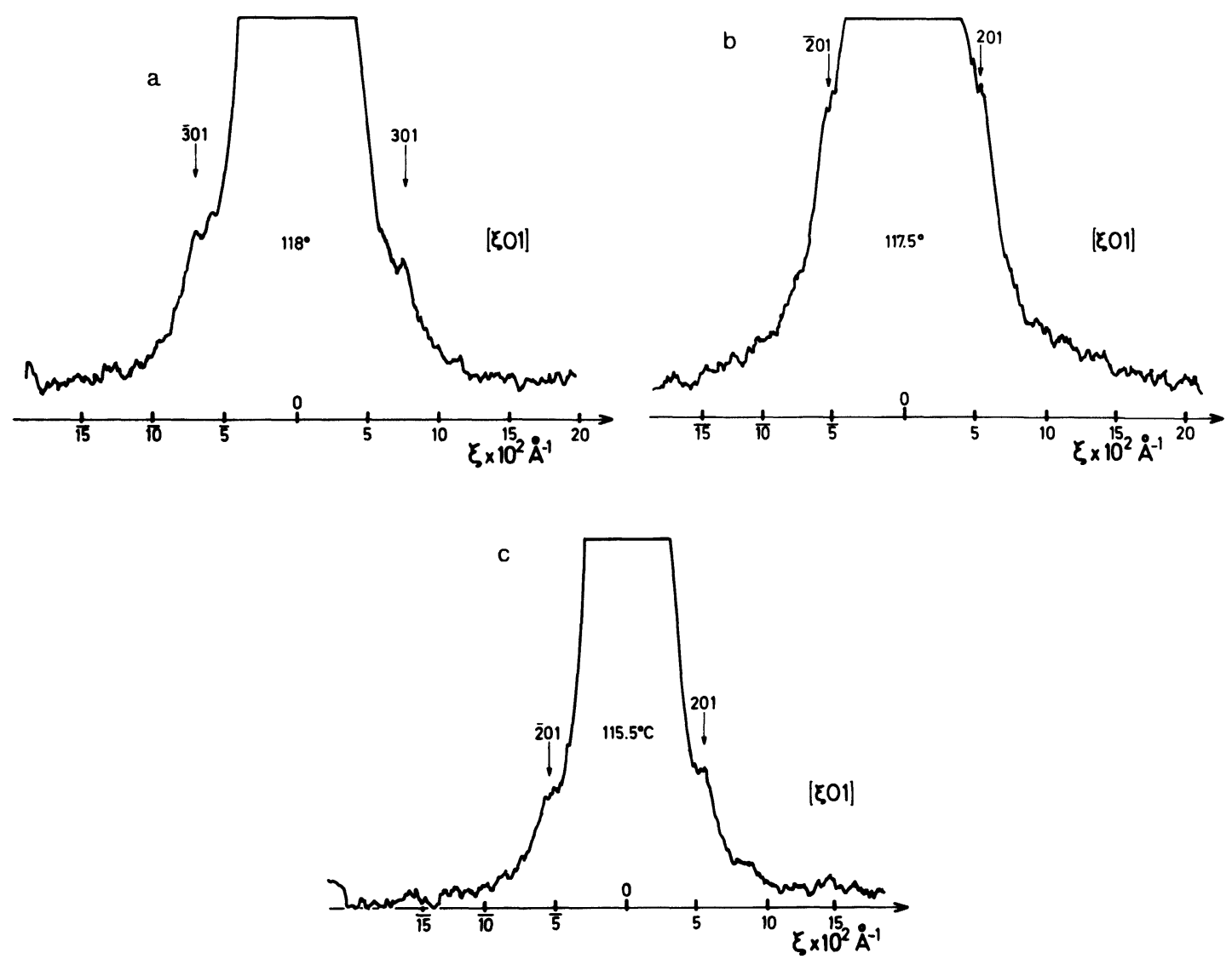

Fig. 4. - $q$ scans of the first layer line parallel to the layer plane in the rectangular phases. a) $118^{\circ} \mathrm{C}$ centred rectangular lattice; b) $117.5^{\circ} \mathrm{C}$ and c) $115.5^{\circ} \mathrm{C}$ rectangulàr lattice.

find alternatively nitriles and aliphatic ends localized in parallel stripes of equal width. Two successive interfaces are in opposite phases, nitrile ends being in front of aliphatic ends and vice versa. This structure can also be described by the juxtaposition of domains with $\mathrm{S}_{\mathrm{A}_{2}}$ structure of infinite dimensions along the director and in one normal direction and of width $a / 2$. Two adjacent domains are translated one to the other by a vector $\frac{1}{2} \mathbf{c}$. Therefore each domain is the analog of the antiphase domains which are observed in some alloys [4].

Two remarks can be made. In this simple description no deformation (undulation) of the layerplane takes place and it is coherent with the absence of reflections $h 0 l, l=2 n, h \neq 0$.

In this model we have assumed that the modulation of the orientation of the dipoles inside a layer had a square form while experimentally the modulation seems to be nearly sinusoidal. One must think that the perfectly ordered zones are narrow; the space between these ordered zones is filled up by a disordered $S_{A_{1}}$ type material. In other words, if we look inside a layer parallel to $X$, we will find a $S_{A_{2}}$ region progressively transforming into a $S_{A_{1}}$ region and then into a $S_{A_{2}}$ region of the opposite phase (Fig. 6a). As we approach the $S_{A_{2}}$ phase, the disordered regions diminish in size inducing higher order reflections. At the same time the period of the modulation (the distance between two antiphase walls) increases. At a certain temperature this distance reaches its maximum value and the centre of the rectangular lattice becomes different from the corner, inducing reflections $h 0 l$ with $h+l=2 n+1$. 


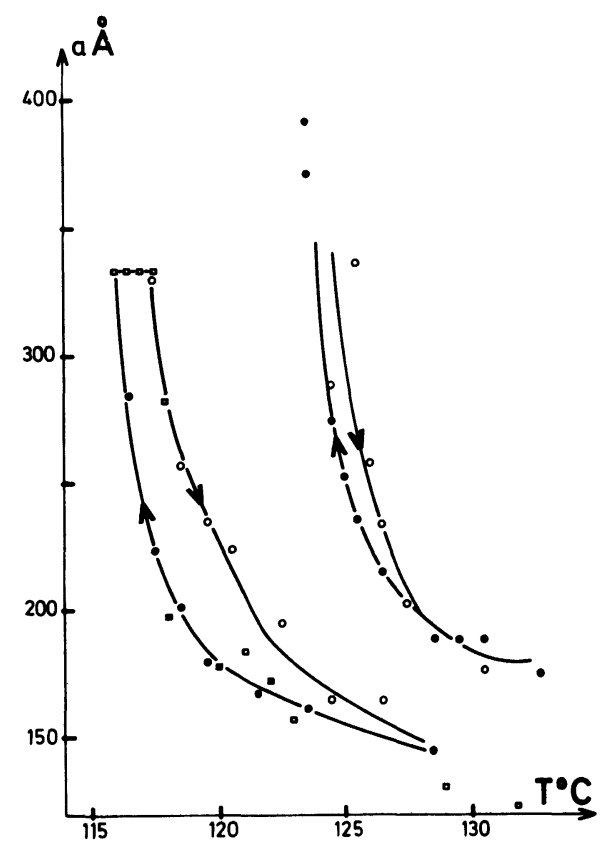

Fig. 5. - Temperature dependence of the period parallel to the smectic planes in the rectangular phases; in the left part a sample with $55 \%$ mole of $\mathrm{DB}_{6}$ with a Nematic- $\mathrm{S}_{\mathrm{A}_{1}}$ transition temperature of $157^{\circ} \mathrm{C}$; in the right part a sample richer in $\mathrm{DB}_{6}\left(\mathrm{Nematic}_{-\mathrm{S}_{\mathrm{A}_{1}}}\right.$ transition at $\left.153.5^{\circ} \mathrm{C}\right)$. ( $(\boldsymbol{a})$ single domain experiments with decreasing temperature; ( $\square$ ) single domain experiment with increasing temperature; (๑) small angle scattering experiments with decreasing temperature $;(O)$ small angle scattering experiments with increasing temperature; the full lines are only guides for the eye.

We must notice that there is no longer any evidence of $h 0 l$ reflections with $h \neq 0$ and $l=2 n$. Therefore we have to keep the layer out of deformation and we must suppose that one kind of antiphase domains becomes narrower while the other kind increases. This alternating of ribbons of unequal width occurs in a large zone of the sample since the Bragg peaks remain sharp(Fig. $6 \mathrm{~b}$ ) and the $\mathrm{S}_{\mathrm{A}_{2}}$ phase corresponds to the annihilation of one kind of the antiphase domains.

If we assume that the walls between two domains are thin and that the proportion of the antiphase domains changes without a change in their internal organization, the ratio of the intensities of reflections 001 and 002 depends on $m$, the proportion of the two kinds of antiphase domains in the lattice

$$
\frac{I(001)}{I(002)}=\left(\frac{I(001)}{I(002)}\right)_{\mathrm{S}_{\mathrm{A}_{2}}} \times(1-2 m)^{2}
$$

We have measured this ratio by using the reflections 001 and 002 at a wavelength of $\frac{1.74}{3} \AA$, which is selected by the Ge 111 crystal, but has a very low intensity; values of $m$ are reported on figure $7 ; m$ varies from 0 to 0.5 almost continuously.

The behaviour of antiphase domains in this fluid system is different from the behaviour of the antiphase domains in alloys [4]. The period and the proportion of the two kinds of domains both have a greater amplitude of variation and this difference is probably due to the fluid character of the mesophases. Since no periodic order of short period is present inside the layers, the long period can vary continuously and its value is probably driven by a balance between elastic and 

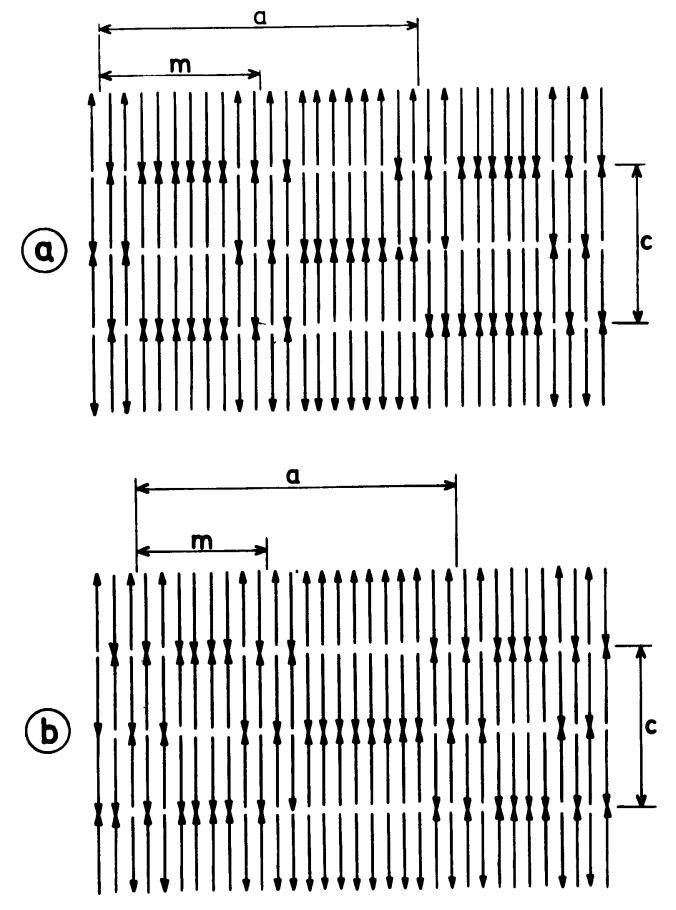

Fig. 6. - Structural models of the two rectangular phase, $a$ and $c$ are the lattice spacings perpendicular and parallel to the director. $m$ is the relative amount of one kind of antiphase domains; a) centred rectangular phase; b) rectangular phase.

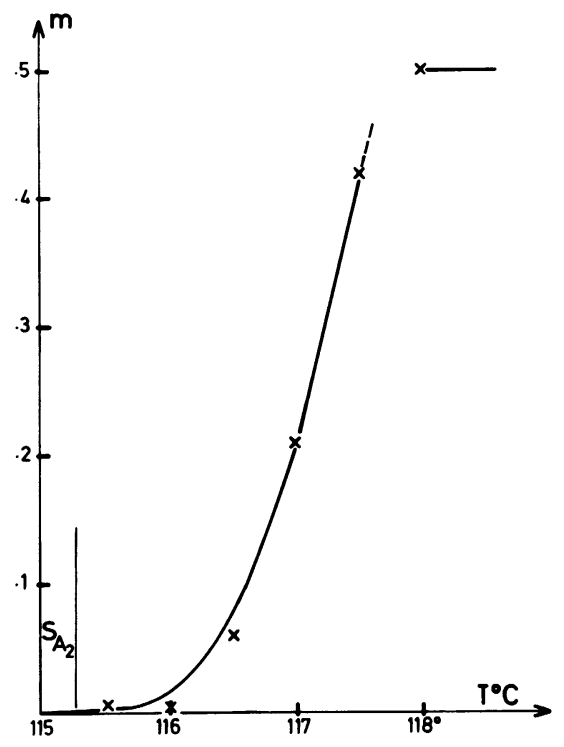

Fig. 7. - Temperature dependence of the proportion of one kind of antiphase domain in the rectangular phase. 
dipolar forces. In this context, the existence of a plateau in the dependence of the long period is surprising. The " lock-in » of the long period implies a break in the symmetry in which one kind of antiphase domain becomes dominant.

The hysteresis observed in the temperature dependence of the long period (distance between two neighbouring walls) is not surprising and appears to be related to the existence of the « lockin " periodicity.

Finally, we have to underline the importance of defects in these organizations. Because of the fluidity of these phases, an easy diffusion of the molecules can favour the evolution of the lattice size and the phases transformation. We have to notice that diffuse scattered intensity can be observed, localized on $0 \mathrm{hl}$ planes of the two rectangular phases. This diffuse scattered intensity can be interpreted either by the existence of columns of molecules with an antiparallel array of dipoles along their axes which are out of phase with respect to the positions defined by the mean structure, or by the existence of soft undulation modes of the layer, with a phase shift of $\pi$ between adjacent monomolecular layers. (In fact, the difference between these two explanations is mainly a matter of language.)

\section{Conclusion.}

In our high resolution study of single domains of Smectic $A$ phases $\mathrm{DB}_{6}-\mathrm{C}_{5}$ mixtures we have shown a symmetry change in the two-dimensional ribbon structure : the centred rectangular phase can transform itself into a simple rectangular phase. We go from an array of two equally important kinds of antiphase domains toward a situation in which one kind of antiphase domains becomes dominant. In the first step, the distance between antiphase walls increases while in the second step the distance between two equivalent walls remains constant, and the proportion of the two kinds of antiphase domains lying between them evolves until we reach a totally ordered structure.

\section{Acknowledgments.}

I am indebted to M. F. Achard and Nguyen Huu Tinh from CRPP (Talence) for the preparation of the sample. The single crystal experiments have been done at LURE with the help of S. Megtert to whom I am grateful. I thank the Laboratoire de l'Accélérateur Linéaire for their continued help and I am indebted to T. Simonson for reading the English manuscript.

\section{References}

[1] Hardouin, F., Levelut, A. M., Achard, M. F., Sigaud, G., J. Chim. Phys. 80 (1983) 53.

[2] Hardouin, F., Levelut, A. M., Benattar, J. J., Sigaud, G., Solid State Commun. 33 (1980) 337.

[3] Sigaud, G., Hardouin, F., Achard, M. F., Levelut, A. M., J. Physique 42 (1981) 107.

[4] Sato, H., Tотн, R. S., "Alloying behaviour and effect in concentrated solid solution ", T. B. Massaki ed. (Gordon and Breach London), Metall. Soc. Conf. (Proc.) 29 (1963) 295. 